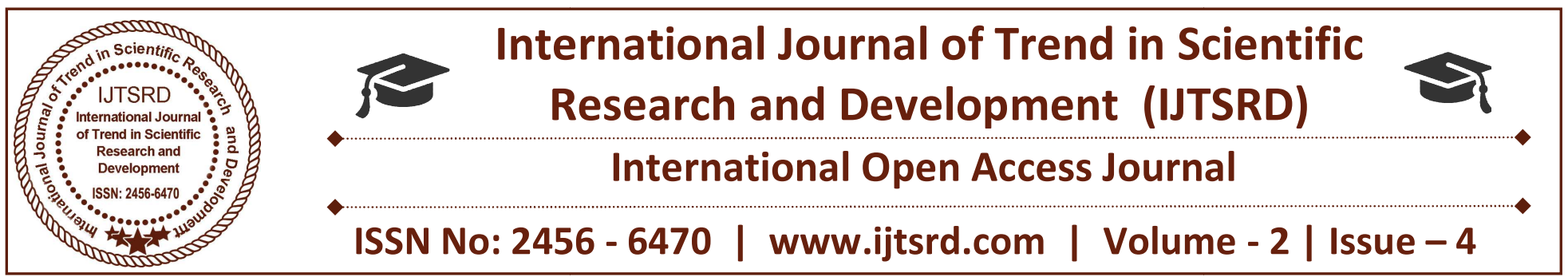

\title{
HMM-Based Face Recognition System with SVD Parameter
}

\author{
Neha Rana ${ }^{1}$, Bhavna Pancholi ${ }^{2}$ \\ ${ }^{1}$ PG Student, ${ }^{2}$ Assistant Professor, \\ Department of Electrical Engineering, Faculty of Technology \& Engineering, \\ The M. S. University, Baroda, Gujarat, India
}

\begin{abstract}
Today an increasing digital world, personal reliable authentication has become an important human Computer interface activity. It is very important to establish a person's identity. In today existing security mainly depends on passwords, swipe cards or token based approach and attitude to control access to physical and virtual spaces passport. Universal, such as methods, although very secure. Such as tokens, badges and access cards can be shared or stolen. Passwords and PIN numbers can be also stolen electronically. In addition, they cannot distinguish between authentic have access to or knowledge of the user and tokens. To make a system more secure and simple with the use of biometric authentication system such as face and hand gesture recognition for personal authentication. So in this paper, A Hidden Markov Model (HMM) based face recognition system using Singular Value Decomposition (SVD) is proposed.
\end{abstract}

Keywords: Face Recognition, Hidden Markov Model (HMM), Singular Value Decomposition (SVD)

\section{INTRODUCTION}

Existing security measures an knowledge-based approaches like passwords or token-based approaches such as swipe cards and passports to control access to physical and virtual spaces. Though ubiquitous, such methods are not very secure. Tokens such as badges and access cards may be shared or stolen. Passwords and PIN numbers may be stolen electronically. Even they cannot differentiate between authorized user and a person having access to the tokens or knowledge. For that face recognition system is very useful.

Biometrics such as fingerprint, face and voice print offers means of reliable personal authentication that can avoid these problems. Biometrics is more reliable system than token or knowledge based authentication methods. The various biometric modalities can be broadly categorized as,

1. Physical Biometric which involve some form of physical measurement such as face, fingerprints, iris-scans, hand geometry etc

2. Behavioural biometrics which are usually temporary in nature and involve measuring the way in how user performs certain tasks like speech, signature, gait etc.

3. Chemical biometrics, This is still a nascent field and involves measuring chemical cues such as chemical composition of human perspiration.

In Human already they have internal skill to recognize different faces. Face recognition is the recognizing a special face from a set of different faces. Face has a significant role in human communications where, each person mainly distinguished by his/her face image. One can easily find out that one of the main problems in machine-human being interactions is the face recognition problem. A human face is a complex object with features varying over time. So a robust face recognition system must operate under a different conditions. Face recognition article guides us step wisely from experts. Face recognition system has many application like security access control, Human computer Interaction, surveillance etc. 
In this paper we are going to study three stage of authentication,

1. Face Detection

2. Face Recognition

3. Hand Gesture detection and recognition
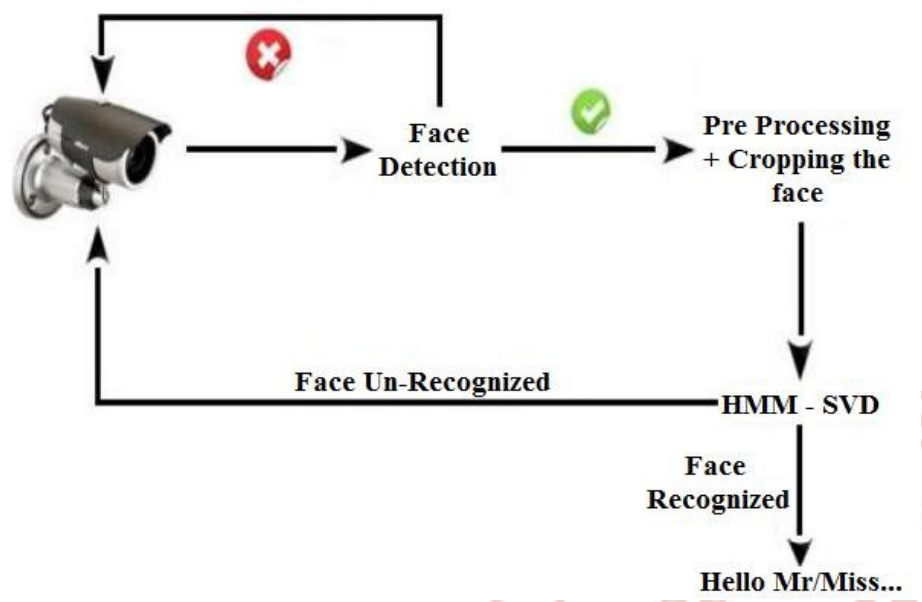

Figure 1 : General block diagram of Face Recognize system

This project will contain some phases, in phase one it detect the face, the camera will be set on video streaming once person is coming it takes a snapshot after detecting the face, in phase two which will be to extract the features necessary to recognize the face, by using Gabor features, phase three will be making the right decision based on a data-base trained models, phase four will make the system say "Hello Mr/Miss....".

\section{LITERATURE REVIEW}

There are some face recognition methods. Some common face recognition methods are Geometrical Feature Matching, Eigen faces method, Neural Networks (NN), Support Vector Machines (SVM), Elastic Matching and Hidden Markov Models (HMM).

The first approach, proposed by Kanade in the 70's, were based on geometrical features. Geometrical Feature Matching techniques are based on the extraction of a set of geometrical features forming the picture of a face. Their system achieved approx 75\% recognition rate on a database of twenty persons, using two images per person: one for training and the other for test. In summary, geometrical feature matching techniques is not a high accurate method and also are rather time-consuming. The other one of the well-known face recognition algorithms, is Eigen faces method. This method uses the Principal Component Analysis (PCA) to project faces into a low dimensional space. This method proved to be not robust against the variations of facial orientation. The authors reported $90.5 \%$ correct recognition on ORL database. In short, Eigen faces method is a fast, simple and practical method. However, it generally does not provide invariance over changes in scale and lighting conditions. Next one, Neural Networks is one of the approaches which have been used in many pattern recognition tasks. The attractiveness of using neural networks could be due to their ability in nonlinear mapping. This had been capable to recognize up to 200 people and could achieve up to $96 \%$ correct recognition rate. In general, neural network approaches encounter problems when the number of face classes increases. The last approach is the stochastic modelling of non-stationary vector time series based on Hidden Markov Models (HMM) which has been widely used in recent years.

\section{METHODOLOGY}

\section{Hidden Markov Model (HMM)}

The Hidden Markov Models are stochastic models which provide a high level of flexibility for modeling the structure of an observation sequence. They allow for recovering the (hidden) structure of a sequence of observations by pairing each observation with a hidden state. Hidden Markov Models (HMMs) used to represent and characterize the statistical property of a signal. They are used not only for speech and handwriting recognition but they are involved in modeling and processing images too. This is the case of their use in the face recognition.

Hidden Markov Models have been successfully used for speech recognition where data is essentially one dimensional. The HMM work on sequences of symbols called observation. Extension to a fully connected two dimensional HMM has been shown to be computationally very complex. For frontal face images, the significant facial regions (hair, forehead, eyes, nose, and mouth) come in a natural order from top to bottom, even if the images are taken under small rotations in the image plane and/or rotations in the plane perpendicular to the image plane. Each of these facial regions is assigned to a state in a left to right 1D continuous HMM.

Below Figure-2 shows signal dimensional HMM for the five segments for face recognition. 


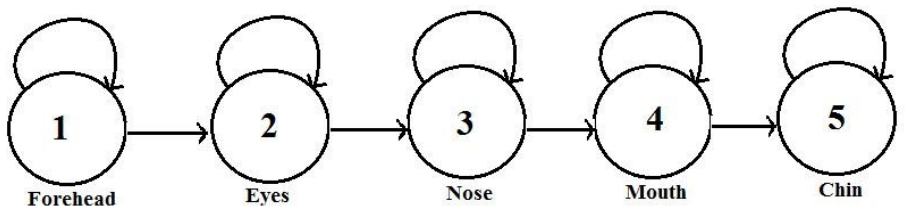

Figure-2.One dimensional HMM with face model

\section{Training and Recognition}

The face images of every subject in the database is identified by a HMM face model. A dataset of five image vectors representation different instances of the same face image are put to train each of the 5-level HMM. The HMM $\lambda=(\mathrm{A}, \mathrm{B}, \pi)$ is initialized. The training data is segmented in $\mathrm{N}=5$ states and the observation vectors associated with each individual state are used to obtain the initial estimation of matrix ' $B$ '. The initial values of matrix ' $A$ ' and ' $\pi$ ' are assumed to given the sliding window structure of the model. The next step is to re-estimate the face model using Expectation Maximum algorithm to increase the probability. This iterative process is terminated after the convergence is achieved. A transform the face image to a uniform by level and size normalized. Then transform the face image to fractal matrix, use the matrix of fractal coding into HMM to calculate the probability. Choose the max matching probability recognition. If all of the matching probability is minor consider as the face image can't find the matching face in this face database.

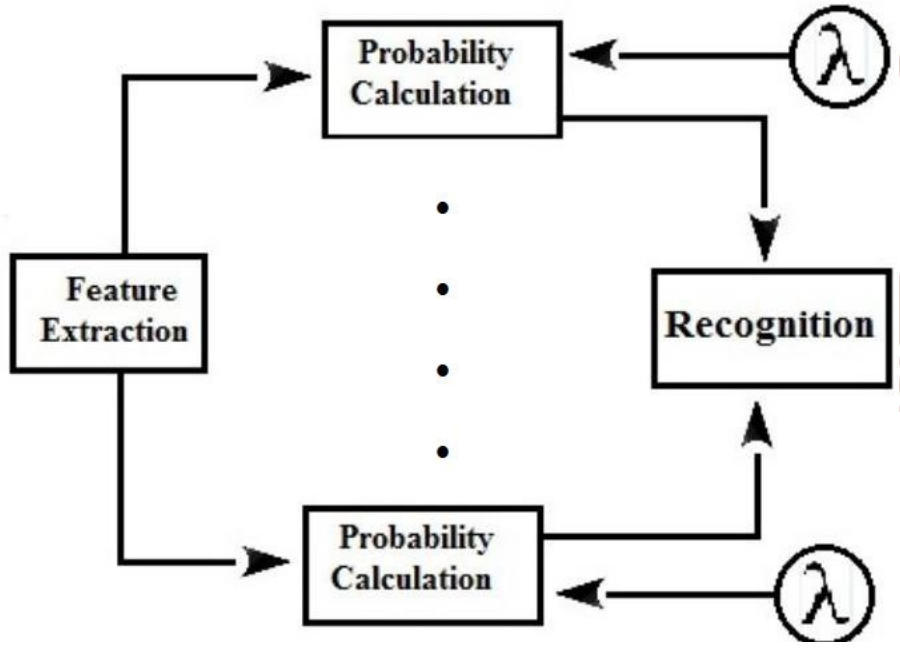

Figure-3: 1-Dimensional HMM for face recognition

\section{Singular Value Decomposition (SVD)}

The Singular Value Decomposition (SVD) has been an important tool in signal processing and statistical data analysis. Singular values of given data matrix contain information about the noise level, the energy, the rank of the matrix, etc. As singular vectors of a matrix are the span bases of the matrix, and orthonormal, they can exhibit some features of the patterns embedded in the signal. Singular Value Decomposition (SVD) provides a new way for extracting algebraic features from an image.

A SVD of a $m \times n$ matrix $X$ is any function of the form:

$\mathrm{X}=$

Where $\mathrm{U}(\mathrm{m} \times \mathrm{m})$ and $\mathrm{V}(\mathrm{m} \times \mathrm{m})$ are orthogonal matrix, and $\mathrm{W}$ is and $\mathrm{m} \times \mathrm{n}$ diagonal matrix of singular values with components $=0, \mathrm{i}=\mathrm{j}$ and $>0$. Furthermore, it can be shown that there exist non-unique matrices $U$ and $\mathrm{V}$ such that $\sigma 1 \geq \sigma 2 \ldots \geq 0$. The columns of the orthogonal matrices $\mathrm{U}$ and $\mathrm{V}$ are called the left and right singular vectors respectively, an important property of $\mathrm{U}$ and $\mathrm{V}$ is that they are mutually orthogonal. The main theoretical property of SVD relevant to face image recognition is its stability on face image. Singular values represent algebraic properties of an image. So because of these reasons and some experimental results, we find out that SVD is a robust feature extraction technique for face images.

Table-1. Comparative result of Different Methods

\begin{tabular}{|l|l|}
\hline $\begin{array}{l}\text { Method (Learning } \\
\text { algorithm) }\end{array}$ & $\begin{array}{l}\text { Percentage of Error } \\
(\%)\end{array}$ \\
\hline $\begin{array}{l}\text { Pseudo 2D HMM + Gray } \\
\text { tone }\end{array}$ & $5 \%$ \\
\hline Sliding HMM + Grey tone & $13 \%$ \\
\hline Eigenface & $10 \%$ \\
\hline Gabor filter + rank & $9 \%$ \\
\hline EBGM & $<20 \%$ \\
\hline $\begin{array}{l}\text { Continuous } \\
\text { classifier }\end{array}$ & $3 \%$ \\
\hline Up-Down HMM + DCT & $16 \%$ \\
\hline Markov Random Fields & $13 \%$ \\
\hline SVM+PCA & $3 \%$ \\
\hline 1D HMM+SVD & $1 \%$ \\
\hline
\end{tabular}


This table shows proposed system has a recognition rate of $99 \%$, a high rate that can simply be approximated to $100 \%$. The significance of this result is that such a high recognition rate is achieved using $\mathrm{HMM}+\mathrm{SVD}$ parameter.

\section{SIMULATION RESULT}

Stage 1: Run code in mat-lab file. This code is made by using HMM+SVD algorithm.

Stage 2 : After running the code it will show one box which ask to train face of employee.

$\mathrm{HMM}+\mathrm{SVD}$ technique is used because in this error ratio is only $1 \%$ comparing to other system. And rate of recognition is about to $99 \%$.

Here it works in two phase,

a)Training

b)Face Recognition

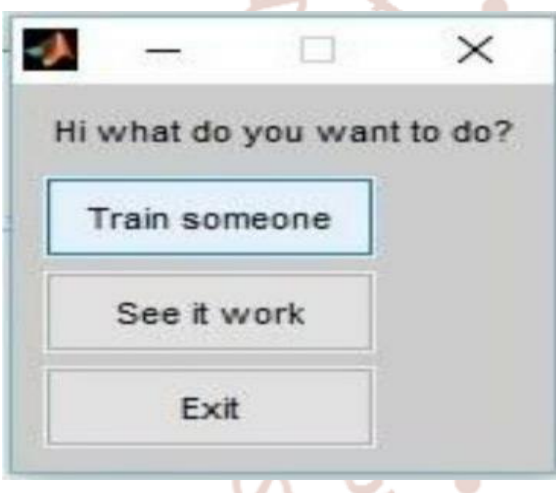

Figure-3: Train Person

Stage 3: Enter the employee's name.

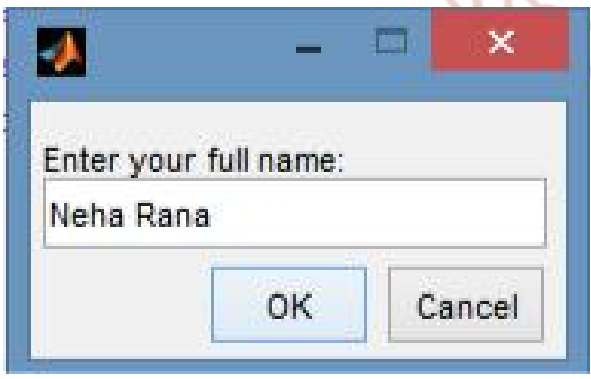

Figure-5: Enter Person's name

Stage 4: Now it will take up to 400 photographs of different angles of face in 5 seconds. And ask for train these photographs.

For face detection we use Viloa Jones algorithm. It detects only faces. It create frame towards face. It only detects face, but not recognize. Here different types of algorithms are available but

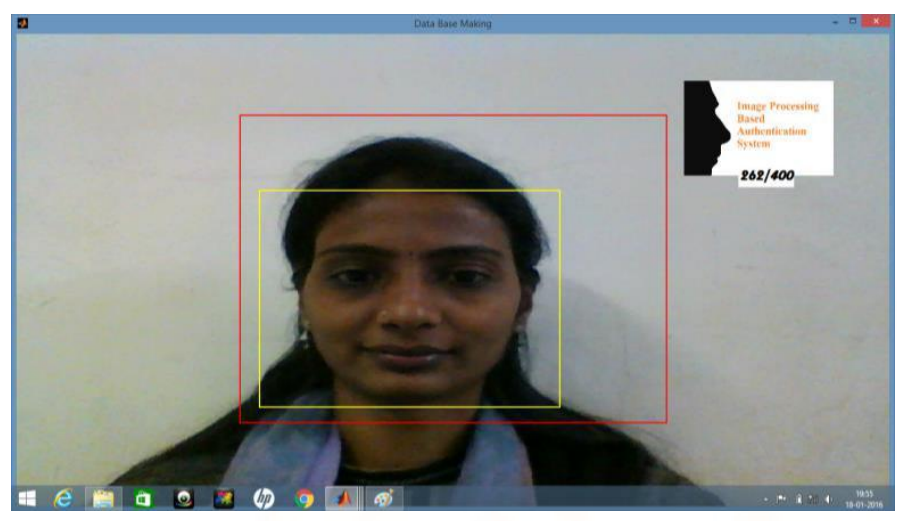

Figure-6: Image Capturing

Stage 5 : For first time training the 400 photographs it will take around 12 to 15 minutes. As soon as training of photographs was completed, data base is ready of that employee. And it will show message of training were done using SVD parameter. It will save all data in .mat type database file.
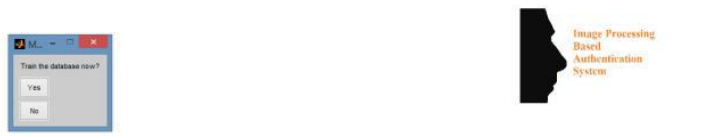

Jhank You

Figure-7: Train Person`s Face Image Database

Stage 6: After training the face system is ready for the work. It will ask for see the work.

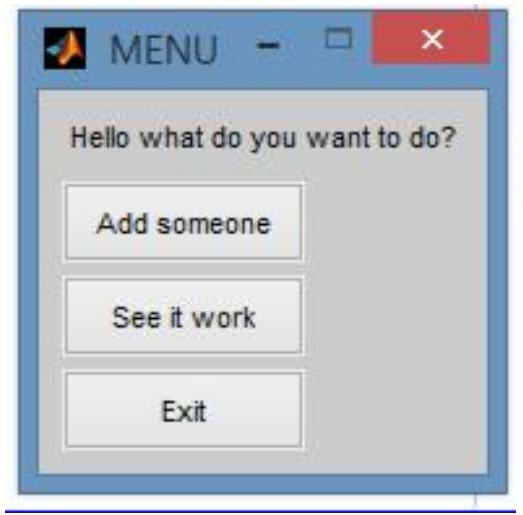

Figure-8: See it Works 
Stage 7: When person will come in default area, camera automatically captures the image of person and try to recognize person from their database and show the message "Hello Mr/miss...". If person is outside of the default area or in between that it will show "No Person Found".

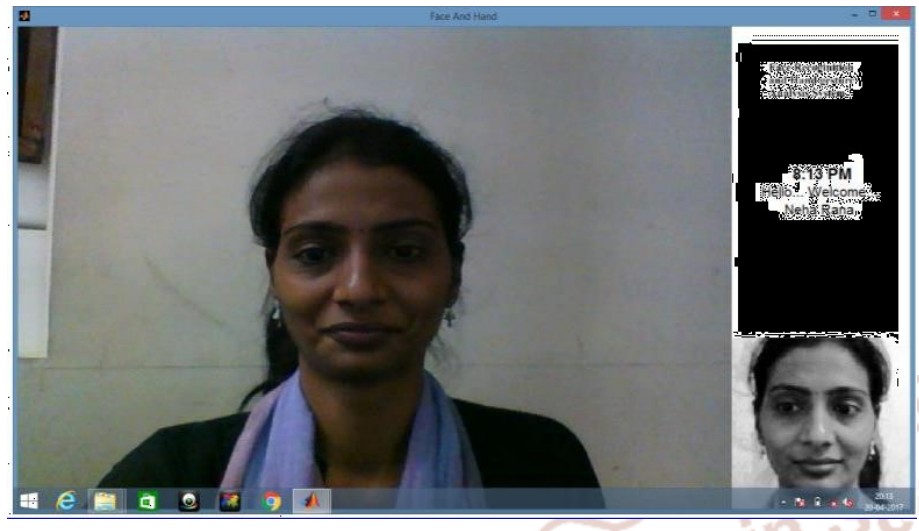

Figure-9: Face Recognition

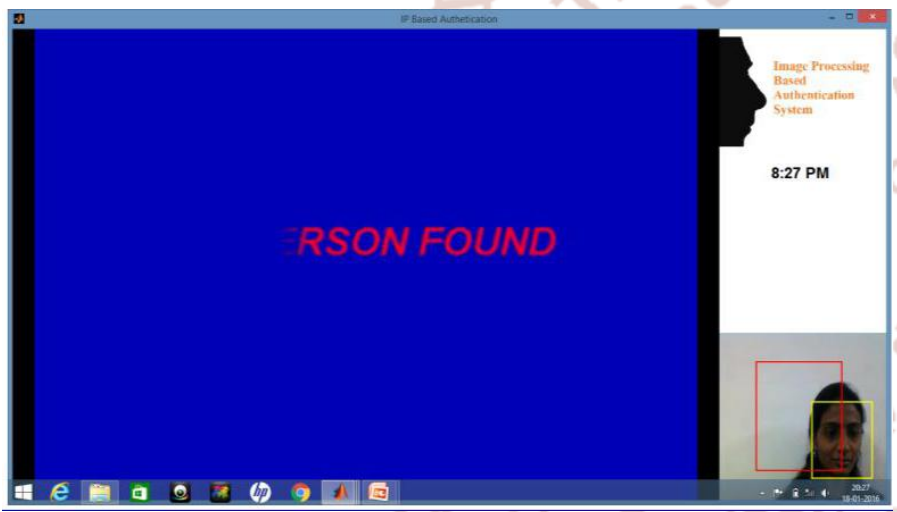

Figure-10: Face outside the default area

\section{CONCLUSION}

In the above system, we discussed about to make a system more secure and simple with the use of biometric authentication with the help of HMM algorithm with SVD parameters. We have categorically achieved our goal of recognizing face image from a large dataset with few set of training images. The experiments carried out indicate that, the HMM work efficiently when there is more learning detail and takes more time to recognize. In this system, approximately having a recognition rate of $99 \%$, the system was very fast.

This system could be useful in E-Government services, National Security, Airport, Bank, Offices, colleges, Biometric Time Attendance System.

\section{REFERENCES}

1) Rowayda A. Sadek "SVD Based Image Processing Applications: State of The Art, Contributions and Research Challenges", ,(IJACSA) International Journal of Advanced Computer Science and Applications, Vol. 3, No. 7, 2012

2) Mukundhan Srinivasan and Sriram Raghu "Comparative Study on Hidden Markov Model versus Support Vector Machine A ComponentBased Method for Better Face Recognition”, 2013 UKSim 15th International Conference on Computer Modelling and Simulation.

3) S. Tolba, A.H. El-Baz, and A.A. El-Harby "Face Recognition: A Literature Review", International Journal of Signal Processing 2;2 2006

4) H. Miar-Naimi and P. Davari "A New Fast and Efficient HMM-Based Face Recognition System Using 7-State HMM Along With SVD Coefficients", Iranian Journal of Electrical \& Electronic Engineering, Vol. 4, Nos. 461 \& 2, Jan. 2008

5) Mukundhan Srinivasan, Nivas Ravichandran "A New Technique For Face Recognition Using 2DGabor Wavelet Transform With 2D-Hidden Markov Model fi Approach", 978-1-4673-4862-1/13/\$31.00 C2013 IEEE

6) A. Pentland, B. Moghadam, T. Starner and M. Truk, "View based and modular eignspaces for face recognition," in Proceedings on IEEE Computer Society Conference on Computer Vision and Pattern Recognition, pp. 84-90, 1994.

7) Arpita Ray Sarkar G. Sanyal S. Majumder "Hand Gesture Recognition Systems: A Survey", , International Journal of Computer Applications (0975 - 8887) Volume 71- No.15, May 2013

8) R. Chellapa, C. Wilson, S. Sirohey, Human and machine recognition of faces: a survey, Proc. IEEE 83, No. 5, Vol 5 May 1995pp. 705740.

9) A. S. Tolba, A.H. El-Baz, and A.A. El-Harby "Face Recognition: A Literature Review"

10) PETYA DINKOVA1, PETIA GEORGIEVA2, MARIOFANNA MILANOVA3 "Face recognition using Singular Value Decomposition and Hidden Markov Models" 